\title{
Estimation of reactor neutrino fluxes
}

\section{Daniel A. Dwyer*}

Lawrence Berkeley National Laboratory

E-mail: dadwyer@lbl.gov

Reactor antineutrinos have been indispensable for our understanding of neutrino mass and mixing. At the same time, discrepancies between the observed and predicted reactor $\bar{v}_{e}$ rate and energy spectra have grown as the precision of these measurements has improved. Measurements of the electrons emitted following fission result in the most precise predictions for the corresponding $\bar{v}_{e}$ flux, and our understanding of the potential systematic differences between the fission $e^{-}$and $\bar{v}_{e}$ fluxes has improved. Measurements of individual fission daughter isotopes and their decays are fraught with uncertainties, yet still provide insight into these discrepancies. Detailed comparisons of $\bar{v}_{e}$ measurements among reactors are also shedding new light on this topic.

Neutrino Oscillation Workshop

4 - 11 September, 2016

Otranto (Lecce, Italy)

${ }^{*}$ Speaker. 


\section{Introduction}

Antineutrinos emitted by nuclear fission reactors have served as a powerful tool for the study of these weakly-interacting particles. The intense flux from reactors, roughly $10^{20} \bar{v}_{e}$ per second per $\mathrm{GW}_{\text {th }}$ of reactor power, was used for the first detection of these elusive particles [1]. Measurements of reactor antineutrinos have also revealed the distinct signature of the oscillation of neutrino flavor [2,3]. On the other hand, precise models of reactor $\bar{v}_{e}$ emission do not agree with these measurements. The predicted rate is $6 \%$ higher than that observed, a feature that is commonly referred to as the reactor antineutrino anomaly and has been considered possible evidence for sterile neutrinos [4]. More recently, precise measurements of $\bar{v}_{e}$ energy spectra have also shown a $\sim 10 \%$ excess relative to prediction in the region of 5 to $7 \mathrm{MeV}[5,6,7]$. In these proceedings I will examine the details behind these discrepancies, and discuss the substantial recent developments in this field.

The process of reactor $\bar{v}_{e}$ production is well understood. Fission of actinides, in particular ${ }^{235} \mathrm{U},{ }^{238} \mathrm{U},{ }^{239} \mathrm{Pu}$, and ${ }^{241} \mathrm{Pu}$, produce unstable neutron-rich fission fragments. These fission daughter isotopes undergo successive $\beta$-decays until reaching stability, with an average of 6 decays per initial fission. The total $\bar{v}_{e}$ emission from a reactor, $S\left(E_{\bar{v}}\right)$, is the sum of the $\bar{v}_{e}$ 's emitted by these decays,

$$
S\left(E_{\bar{V}}\right)=\sum_{i=0}^{n} R_{i} \sum_{j=0}^{m} f_{i j} S_{i j}\left(E_{\bar{V}}\right)
$$

where $R_{i}$ is the rate of decays of the $i$ 'th fission daughter isotope, $f_{i j}$ is the relative probability for the $j$ 'th decay mode of this daughter (also referred to as the branching fraction), and $S_{i j}\left(E_{\bar{v}}\right)$ is the $\bar{v}_{e}$ energy spectrum for the $j$ 'th decay mode. There are more than 1300 known fission daughter isotopes, and combined they include more than 10,000 unique decay modes.

\section{Current measurements}

Three sets of measurements are particularly relevant to the assessment of reactor $\bar{v}_{e}$ production:

1. direct measurements of reactor $\bar{v}_{e}$ emission,

2. measurements of electron emission following fission, and

3. measurements of the fission yields and decay modes of fission daughters.

Direct measurements commonly involve $\bar{v}_{e}$ detection via inverse beta decay (IBD) in large organic scintillator detectors. Calorimetry of the positrons produced by IBD allow accurate estimation of the rate and energy spectra of those $\bar{v}_{e}$ with energies above the interaction threshold of $1.8 \mathrm{MeV}$. Subsequent detection of the neutrons produced by IBD allows for effective background rejection. The most recent generation of direct measurements have observed more than 1 million $\bar{v}_{e}$ interactions, and obtained percent-level uncertainties in both the rate and energy spectra $[5,6,7]$. This precision has been putting pressure on the field to obtain more accurate predictions of the reactor $\bar{v}_{e}$ flux. 
The most precise predictions have been based on corresponding measurements of the rate and energy spectra of electrons emitted following fission. Due to the kinematic symmetry of the electrons and $\bar{v}_{e}$ produced in $\beta$-decay, their rate and energy spectra are highly correlated. The fission electron spectra were measured at the $2 \%$-level in a series of experiments at the ILL research reactor in Grenoble in the 1980's $[8,9,10,11]$. In these measurements, foils of actinides ${ }^{235} \mathrm{U}$, ${ }^{239} \mathrm{Pu},{ }^{241} \mathrm{U}$ ) were exposed to the neutron flux in the ILL reactor and the emitted electrons were measured. By measuring the cumulative electron spectra due to all the fission daughters and their decay modes, one avoids the need to know the detailed aspects of each daughter. Modeling the electron spectrum as the sum of a large number of $\beta$-decays, the corresponding $\bar{v}_{e}$ spectrum can be calculated [12]. Nuclear corrections to $\beta$-decay do introduce slight asymmetries between the electron and $\bar{v}_{e}$ spectra, as summarized in [13]. A hybrid approach that uses data on fission daughters to inform these nuclear corrections gives a similar result [14]. Overall, this $\beta$-conversion approach provides a prediction for the reactor $\bar{v}_{e}$ rate and energy spectra with uncertainties at the 3\%-level, and has served as the de-facto standard for the past thirty years.

Unfortunately, the direct $\bar{v}_{e}$ measurements and $\beta$-conversion predictions disagree on both the rate and energy spectra, as discussed in the introduction. The origin of these discrepancies are unclear, although potential explanations have been explored [15]. Antineutrinos from the decay of neutron-activated reactor materials, spectral distortions from forbidden decays, and non-thermal fission of ${ }^{238} \mathrm{U}$ do not seem to be large enough to explain the differences. The energy spectra of the neutrons producing fission in the ILL electron measurements differ slightly from that in the commercial reactors used in the direct $\bar{v}_{e}$ measurements. This could result in a slightly different distribution of fission daughters, which is difficult to rule out as a source for the discrepancies between the electron and $\bar{v}_{e}$ data. Another option could be an unknown systematic in the ILL electron measurements, although this is difficult to confirm given that these are the only set of electron measurements to date.

What guidance can the past century of measurements of nuclear fission and decay provide? These measurements, which are collected in nuclear databases such as ENDF, JEFF, and JENDL, can be used to calculate the $\bar{v}_{e}$ flux according to Eq. 1.1. Examples of such calculations can be found in $[16,17,18,19]$. Given the large uncertainties of such calculations, one might conclude that these databases can provide little guidance. For example, $70 \%$ of the known fission daughters lack decay mode data (although these tend to be those daughters which are rarely produced, and hence only amount to $\sim 6 \%$ of the total fission yield). The fission yield data provided by the various databases are inconsistent with each other, and gross errors have been identified [20]. Decay data are generally only known for the most prominent decay modes, and are susceptible to systematic biases from measurement techniques (e.g. the Pandemonium effect).

Despite these obstacles, the shape of reactor $\bar{v}_{e}$ energy spectrum calculated from the ENDF database is unexpectedly similar to the direct $\bar{v}_{e}$ measurements [19]. This may not be wholly surprising, since the spectral shape seems to be dominated by a small number of prominent fission daughters and decay modes which are well-measured. Many of the uncertainties impact daughters and modes which each contribute at most $1 \%$ of the overall $\bar{v}_{e}$ flux, and hence have little influence on the spectral shape. Consequently, there is potential to improve the calculation of the spectral shape through a targeted program of measurement of the most prominent fission daughters. Unfortunately, the rate calculation will likely continue to suffer from large uncertainties due to the 
cumulative effect of the many rare but poorly known fission daughters.

\section{Looking forward}

A targeted program of measurements of the decay modes of prominent fission daughters is being pursued, and has begun to yield results. In particular, measurements of ${ }^{92} \mathrm{Rb}$ and ${ }^{142} \mathrm{Cs}$ using total absorption spectroscopy have already reduced the largest uncertainties in the calculation of the 5 to $7 \mathrm{MeV}$ discrepant region of the $\bar{v}_{e}$ spectrum [21, 22]. Comparison of the ENDF and JEFF databases suggest another important step will be improved measurements of the fission yields of the most prominent daughters, of which ${ }^{96} \mathrm{Y}$ is the most critical [15]. To directly address the tension between the electron and $\bar{v}_{e}$ measurements, a repeat of the ILL electron measurements is being considered at LANL.

Recent work comparing the direct $\bar{v}_{e}$ measurements between different nuclear reactors has also been fruitful. A global analysis of $\bar{v}_{e}$ rate measurements has shown that the rate discrepancy cannot be attributed solely to the minor fission parents such as ${ }^{239} \mathrm{Pu}$ or ${ }^{238} \mathrm{U}$, and instead shows that ${ }^{235} \mathrm{U}$ electron and $\bar{v}_{e}$ data are in tension [23]. A double ratio of the Daya Bay and NEOS observed over expected $\bar{v}_{e}$ spectra also suggests tension between the ${ }^{235} \mathrm{U}$ electron and $\bar{v}_{e}$ energy spectra [24]. Data from the upcoming generation of short-baseline direct $\bar{v}_{e}$ measurements, such as PROSPECT [25], should continue to elucidate. The impressive precision of recent $\bar{v}_{e}$ measurements also suggests interesting potential for reactor characterization and non-proliferation. In general, our understanding of reactor $\bar{v}_{e}$ emission is advancing rapidly and I expect substantial improvements over the coming years.

I would like to thank the organizers of the 2016 Neutrino Oscillation Workshop for the invitation to come speak on this topic. I owe Patrick Huber, Bryce Littlejohn, and Patrick Tsang for thoughtful discussions on these topics. This work was supported under DOE OHEP DE-AC02$05 \mathrm{CH} 11231$.

\section{References}

[1] F. Reines et al, Detection of the free anti-neutrino, Phys. Rev. 117 (1960) 159.

[2] T. Araki et al, Measurement of neutrino oscillation with KamLAND: Evidence of spectral distortion, Phys. Rev. Lett. 94 (2005) 081801 [hep-ex/0406035].

[3] F. P. An et al, New Measurement of Antineutrino Oscillation with the Full Detector Configuration at Daya Bay, Phys. Rev. Lett. 115 (2015) 111802 [arXiv:1505.03456].

[4] G. Mention et al, The Reactor Antineutrino Anomaly, Phys. Rev. D83 (2011) 073006 [arXiv:1101.2755].

[5] F. P. An et al, Improved Measurement of the Reactor Antineutrino Flux and Spectrum at Daya Bay, [arXiv:1607.05378].

[6] H. Seo et al, Spectral Measurement of the Electron Antineutrino Oscillation Amplitude and Frequency using 500 Live Days of RENO Data, [arXiv:1610.04326].

[7] A. Cabrera et al, Double CHOOZ: first multi-detector results, in proceedings of the XXVIth International Conference on Neutrino Physics and Astrophysics, 2016. 
[8] F. Von Feilitzsch et al, Experimental beta spectra from Pu-239 and U-235 thermal neutron fission products and their correlated anti-neutrinos spectra, Phys. Lett. B118 (1982) 162.

[9] K. Schreckenbach et al, Determination of the anti-neutrino spectrum from U-235 thermal neutron fission products up to 9.5-MeV, Phys. Lett. B160 (1985) 325.

[10] A. A. Hahn et al, Anti-neutrino Spectra From ${ }^{241}$ Pu and ${ }^{239}$ Pu Thermal Neutron Fission Products, Phys. Lett. B218 (1989) 365.

[11] N. Haag et al, Re-publication of the data from the BILL magnetic spectrometer: The cumulative $\beta$ spectra of the fission products of ${ }^{235} U,{ }^{239} \mathrm{Pu}$, and ${ }^{241} \mathrm{Pu}$, [arXiv:1405.3501].

[12] P. Vogel, Conversion of electron spectrum associated with fission into the antineutrino spectrum, Phys. Rev. C76 (2007) 025504 [arXiv:0708.0556].

[13] P. Huber, On the determination of anti-neutrino spectra from nuclear reactors, Phys. Rev. C84 (2011) 024617 [arXiv:1106.0687].

[14] T. A. Mueller et al, Improved Predictions of Reactor Antineutrino Spectra, Phys. Rev. C83 (2011) 054615 [arXiv:1101.2663].

[15] A. C. Hayes et al, Possible origins and implications of the shoulder in reactor neutrino spectra, Phys. Rev. D92 (2015) 033015 [arXiv:1506.00583].

[16] P. Vogel et al, Reactor Anti-neutrino Spectra and Their Application to Anti-neutrino Induced Reactions. II., Phys. Rev. C24 (1981) 1543.

[17] M. Fallot et al, New antineutrino energy spectra predictions from the summation of beta decay branches of the fission products, Phys. Rev. Lett. 109 (2012) 202504 [arXiv:1208.3877].

[18] A. A. Sonzogni et al, Nuclear structure insights into reactor antineutrino spectra, Phys. Rev. C91 (2015) 011301.

[19] D. A. Dwyer and T. J. Langford. Spectral Structure of Electron Antineutrinos from Nuclear Reactors, Phys. Rev. Lett. 114 (2015) 012502 [arXiv:1407.1281].

[20] A. A. Sonzogni et al, Effects of Fission Yield Data in the Calculation of Antineutrino Spectra for U235(n,fission) at Thermal and Fast Neutron Energies, Phys. Rev. Lett. 116 (2016) 132502.

[21] A. A. Zakari-Issoufou et al, Total Absorption Spectroscopy Study of ${ }^{92}$ Rb Decay: A Major Contributor to Reactor Antineutrino Spectrum Shape, Phys. Rev. Lett. 115 (2015) 102503 [arXiv:1504.05812].

[22] B. C. Rasco et al, Decays of the Three Top Contributors to the Reactor $\bar{v}_{e}$ High-Energy Spectrum, ${ }^{92} \mathrm{Rb},{ }^{96 g s} \mathrm{Y}$, and ${ }^{142} \mathrm{Cs}$, Studied with Total Absorption Spectroscopy, Phys. Rev. Lett. 117 (2016) 092501 .

[23] C. Giunti, Precise determination of the ${ }^{235}$ U reactor antineutrino cross section per fission, Phys. Lett. B764 (2017) 145. [arXiv:1608.04096].

[24] P. Huber, The 5 MeV bump - a nuclear whodunit mystery, [arXiv:1609.03910].

[25] J. Ashenfelter et al, The PROSPECT Physics Program, J. Phys. G43 (2016) 113001 [arXiv:1512.02202]. 